\title{
Clinical and endoscopic evaluation of dyspeptic patients attending a tertiary care hospital in South India: A prospective study
}

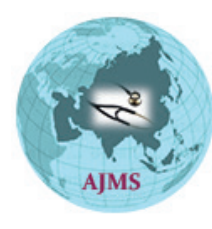

\author{
Robin George Manappallil', Thomas Alexander ${ }^{2}$ \\ ${ }^{1}$ Assistant Professor, Department of Medicine, 2Senior Consultant and Head, Department of Gastroenterology, \\ Pondicherry Institute of Medical Sciences, Pondicherry, India
}

Background: The incidence of dyspepsia has been increasing all over the world; with some having an organic cause while others have functional dyspepsia. Helicobacter pylori infection is also a contributory factor. Moreover, these dyspeptic patients also show a wide spectrum of symptoms. Aims and Objective: The aim of this study was to clinically and endoscopically evaluate the patients with dyspepsia; and also to assess the prevalence of Helicobacter pylori. Materials and Methods: A prospective study was conducted on 100 dyspeptic patients who were advised an upper gastrointestinal endoscopy based on clinical grounds at Pondicherry Institute of Medical Sciences, Pondicherry, India. Results: Of the 100 dyspeptic patients evaluated in this study, the mean age was 42 years, with $54 \%$ belonging to 20 to 40 years, $35 \%$ to 41 to 60 years and $11 \%$ above 60 years age groups. $61 \%$ were males and $39 \%$ females. They belonged to various occupational groups with majority leading a retired life or as homemakers $(36 \%)$, followed by those with white collar jobs or as students (29\%), unskilled workers (26\%) and skilled workers (9\%). Majority of these patients were non vegetarians (83\%). Smokers and alcoholics comprised of $38 \%$ and $43 \%$ of the subjects respectively; and $31 \%$ had history of NSAID consumption. Epigastric pain and epigastric burning sensation were the main complaints among dyspeptic patients. Heartburn was more common among smokers and alcoholics; and epigastric pain among NSAID users. The main endoscopic abnormalities were erythematous and erosive gastritis (42\% and $21 \%$ respectively), followed by duodenal ulcer $(18 \%)$ and erosive duodenitis $(15 \%)$. Duodenal ulcers were more common among males $(89 \%)$. Duodenitis $(89 \%)$ and duodenal ulcers $(67 \%)$ were mainly seen in alcoholics. $18 \%$ of our study patients had a normal endoscopic finding. Helicobacter pylori was present in $35 \%$ of the subjects. Epigastric pain and epigastric burning sensation were the main symptoms among these patients; with erythematous gastritis being the main endoscopic finding. Conclusion: Dyspepsia was more common among younger age groups. Epigastric pain and epigastric burning sensation were the most common symptoms. Erythematous gastritis followed by erosive gastritis was the common endoscopic abnormality. Some patients also had normal findings on endoscopy. Helicobacter pylori was present in a significant number of patients.

Key words: Dyspepsia, Helicobacter pylori, Gastroesophageal reflux disease
Access this article online

Website:

http://nepjol.info/index.php/AJMS

DOI: 10.3126/ajms.v8i1.15932

E-ISSN: 2091-0576

P-ISSN: 2467-9100

\section{INTRODUCTION}

The term dyspepsia was derived from the Greek words "dys" and "pepse" which means difficult digestion. It is defined as pain or discomfort in the upper abdomen.
Other symptoms include early satiation, postprandial fullness, nausea, vomiting, belching, anorexia, heartburn and regurgitation. ${ }^{1}$ Dyspepsia was first recorded in Scotland in the mid-eighteenth century. ${ }^{2}$ In today's world, more than one fourth of the general population suffer from 
dyspepsia. Uninvestigated dyspepsia account for 10 to $40 \%$ of these cases. ${ }^{3}$ Nearly $40 \%$ of dyspeptic patients have an organic cause, which may be structural or physiological. The common causes include peptic ulcer, gastroesophageal reflux disease (GERD) and rarely gastric carcinoma. In more than half of the patients, the aetiology is unknown and is classified as functional dyspepsia. In about $15 \%$ of the patients, the stomach and duodenum is inhabited by a gram negative bacilli called Helicobacter pylori, leading to the development of peptic ulcer disease, with concomitant dyspepsia. ${ }^{1}$

This study aims at evaluating the dyspeptic patients attending our tertiary care hospital, both clinically and endoscopically; and also to assess the prevalence of H. pylori in them.

\section{MATERIALS AND METHODS}

This was a prospective study conducted over a period of 1 year on 100 patients who attended the Gastroenterology department with complaints of dyspepsia at Pondicherry Institute of Medical Sciences, Pondicherry, India. A detailed history was taken and clinical examination was carried out. After obtaining a written and informed consent, the patients underwent upper gastrointestinal (UGI) endoscopy, which was done by a single Gastroenterologist. Two endoscopic mucosal biopsies were taken from the antrum of the stomach for rapid urease test (RUT). All patients with normal endoscopic findings were further advised blood investigations and an ultrasound study of the abdomen to rule out other causes like pancreatic or biliary tree pathology. The endoscope used in this study was Olympus GIF H180 or GIF V70 video endoscope. The study protocol was approved by the Ethics Committee at Pondicherry Institute of Medical Sciences.

\section{Statistical analysis}

The data was analysed using Multiple Logistic Regression Analysis (to determine association between the independent variables like age, sex etc with the dependent variable i.e. endoscopic findings) and Chi Square test (to assess the association of $\mathrm{H}$. pylori in each of these cases).

\section{RESULTS}

Among the 100 dyspeptic patients who were evaluated in this study with ages ranging from 20 to 74 years, the mean age was $42(\mathrm{SD} \pm 14)$ years. The maximum incidence was noted in patients in the age group of 20 to 40 years (54\%), while it was $35 \%$ and $11 \%$ among 41 to 60 years and above 60 years age group respectively.
Off these 100 patients, 61 were males and 39 females. There was no difference between male and female groups with regard to dyspeptic symptoms. The bulk of the subjects comprised of retired people and home makers $(36 \%)$, followed by office workers and students $(29 \%)$. The remaining patients comprised of unskilled $(26 \%)$ and skilled $(9 \%)$ workers. All these patients had an episode of dyspepsia at least once a month over a mean period of 6 (SD \pm 3.6) months. There was no significant difference in the presenting complaints among various occupational groups.

Majority of the patients were non vegetarians (83\%). The type, intensity or frequency of dyspepsia was not related to the type of diet. Out of this, $6 \%$ of these patients had relief of symptoms following food or antacid intake; while $4 \%$ had aggravation of symptoms due to food intake. $10 \%$ of the patients had history of Pantoprazole, Ranitidine or antacid intake as intermittent, over the counter medication. Alcohol consumption was reported in $43 \%$ of our patients, with frequency of 3 to 7 times per week ( 90 to $180 \mathrm{ml}$ ) for a mean duration of 5.3 years. Heartburn was the most common complaint among alcoholics while epigastric burning sensation was more predominant in nonalcoholics. Smokers formed 38\% of the subjects (5-20 cigarettes/ day for the past 5-15 years) and their main symptoms were epigastric pain and epigastric burning sensation. However, there was no difference in the presenting symptoms between smokers and nonsmokers.

Nonsteroidal anti-inflammatory drug (NSAID) intake was noted in $31 \%$ of the patients. The drugs consumed were diclofenac, aceclofenac and ibuprofen. However, information about the duration, dose and frequency of NSAID use were lacking. Epigastric pain was the main complaint among these patients.

Overall, the commonest presenting complaints (Figure 1) were epigastric burning sensation and epigastric pain, found in $97 \%$ and $92 \%$ patients respectively, which were of mild to moderate intensity with a frequency of minimum once a week.

Endoscopic abnormalities were observed in $82 \%$ of these patients (Figure 2), with main findings beingerythematous gastritis $(42 \%)$ and erosive gastritis $(21 \%)$. Erythematous gastritis was mainly found in the corpus of the stomach and they were of mild to moderate severity. Erosive gastritis was of similar severity pattern and was confined to the antrum. Peptic ulcers were found in $22 \%$ of patients; with $18 \%$ having duodenal ulcers and $4 \%$ gastric ulcers. All duodenal ulcers were located in the first part of duodenum and were mostly single ulcer with sizes ranging from 0.5 to $1.25 \mathrm{~cm}$. Duodenal ulcers were more common among males (89\%) 


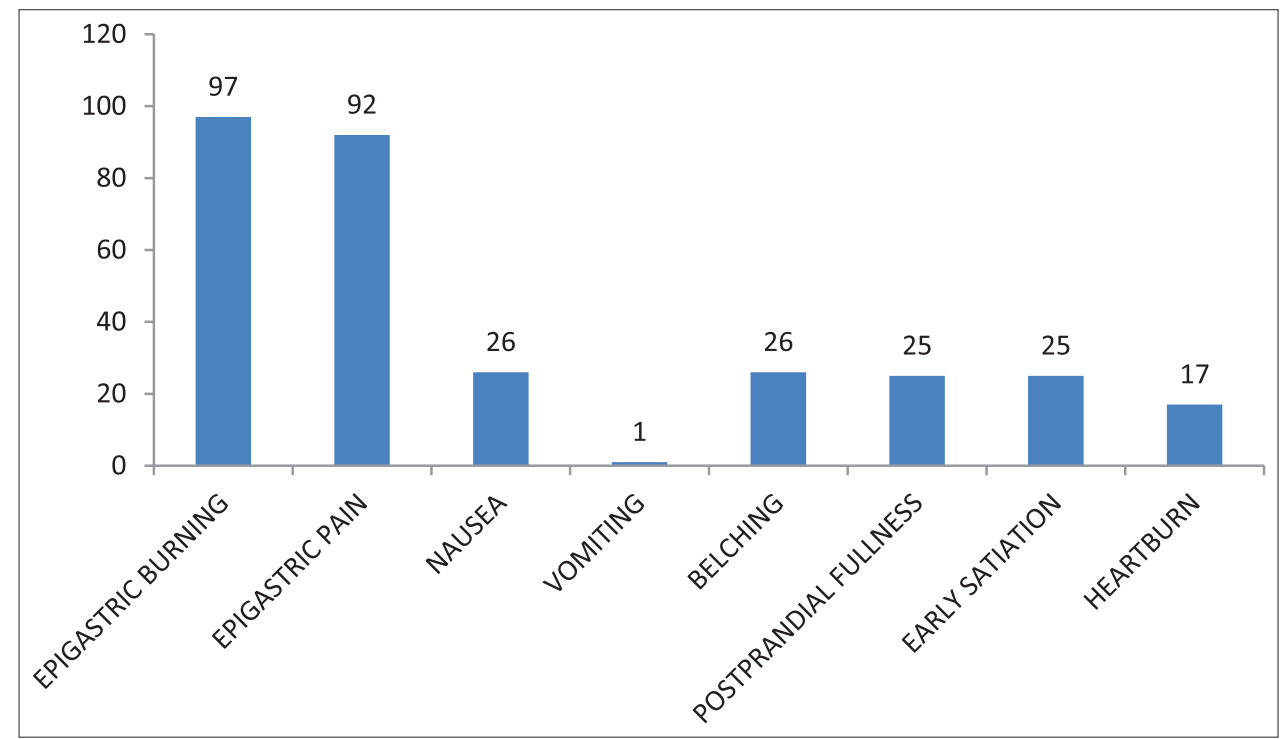

Figure 1: Symptom analysis in dyspepsia

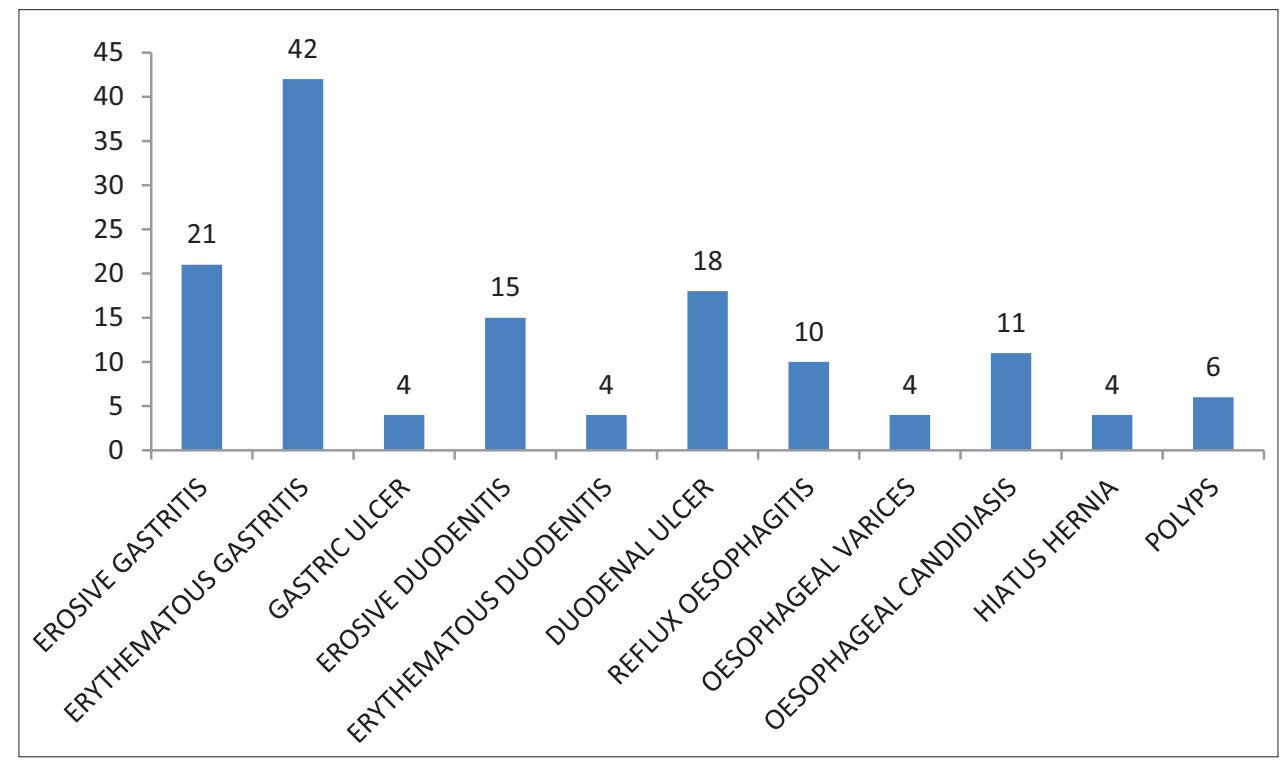

Figure 2: Endoscopic findings in dyspepsia

than females $(\mathrm{P}=0.08)$. Duodenitis was present in $20 \%$ of patients, of which $80 \%$ were erosive and $20 \%$ erythematous. They were of mild to moderate severity and confined to first part of duodenum. Duodenitis and duodenal ulcers were more common among alcoholics $(\mathrm{P}=0.031$ and 0.035 respectively).

UGI endoscopy was normal in $18 \%$ of our patients. The presenting complaints in this group have been depicted in Figure 3. In accordance with the protocol, these patients were further evaluated with liver function tests, serum amylase levels and ultrasound abdomen; which were all found to be normal. No significant correlations were seen between endoscopic findings and age, sex, occupation, diet, drugs or smoking.
In this study, the prevalence of $\mathrm{H}$. pylori was $35 \%$ (detected by RUT). Epigastric pain and epigastric burning sensation were the main symptoms. Erythematous gastritis (51\%) was the most common endoscopic abnormality. Other findings have been depicted in Figure 4. The presence of H. pylori was significantly more among alcoholics $(42 \%, \mathrm{P}=0.036)$; with no significant association with age, sex, occupation, drugs or smoking.

\section{DISCUSSION}

This was a prospective study conducted on 100 dyspeptic patients. They were evaluated clinically and endoscopically. The prevalence of $\mathrm{H}$. pylori was also assessed. 


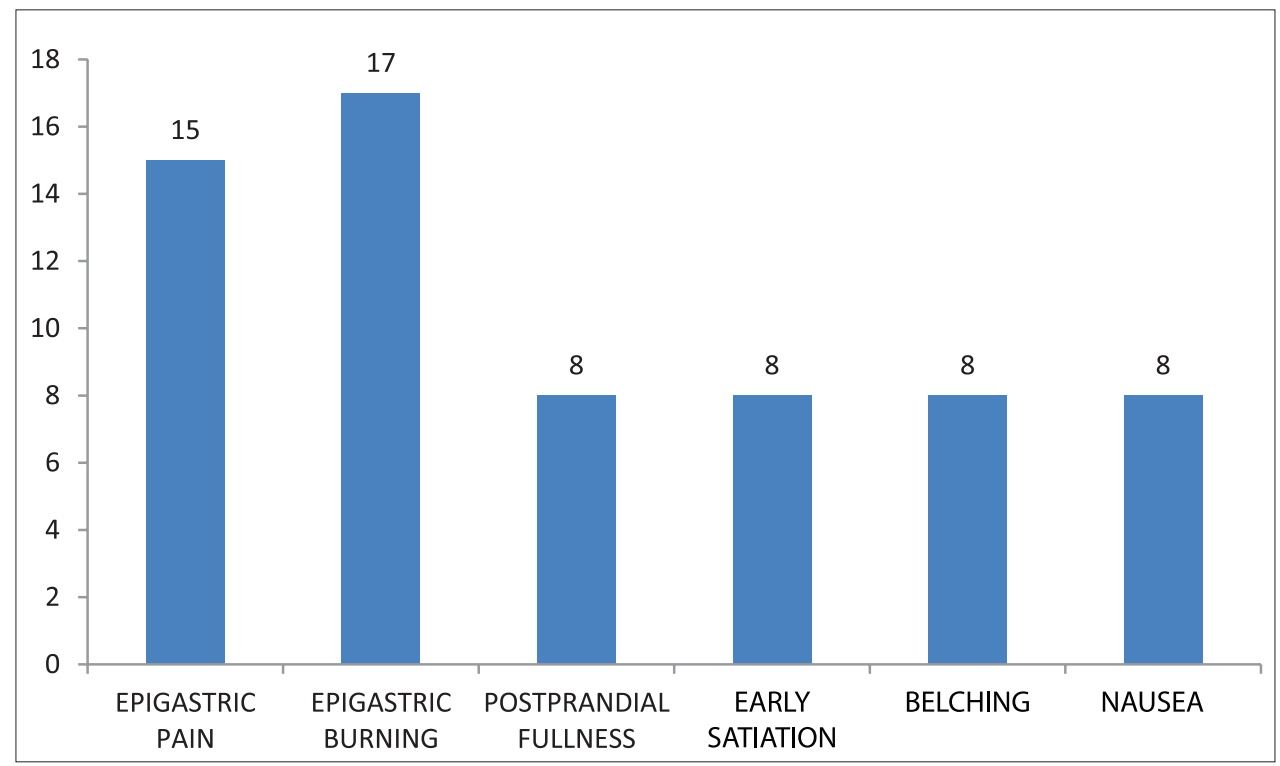

Figure 3: Symptoms in patients with normal endoscopy

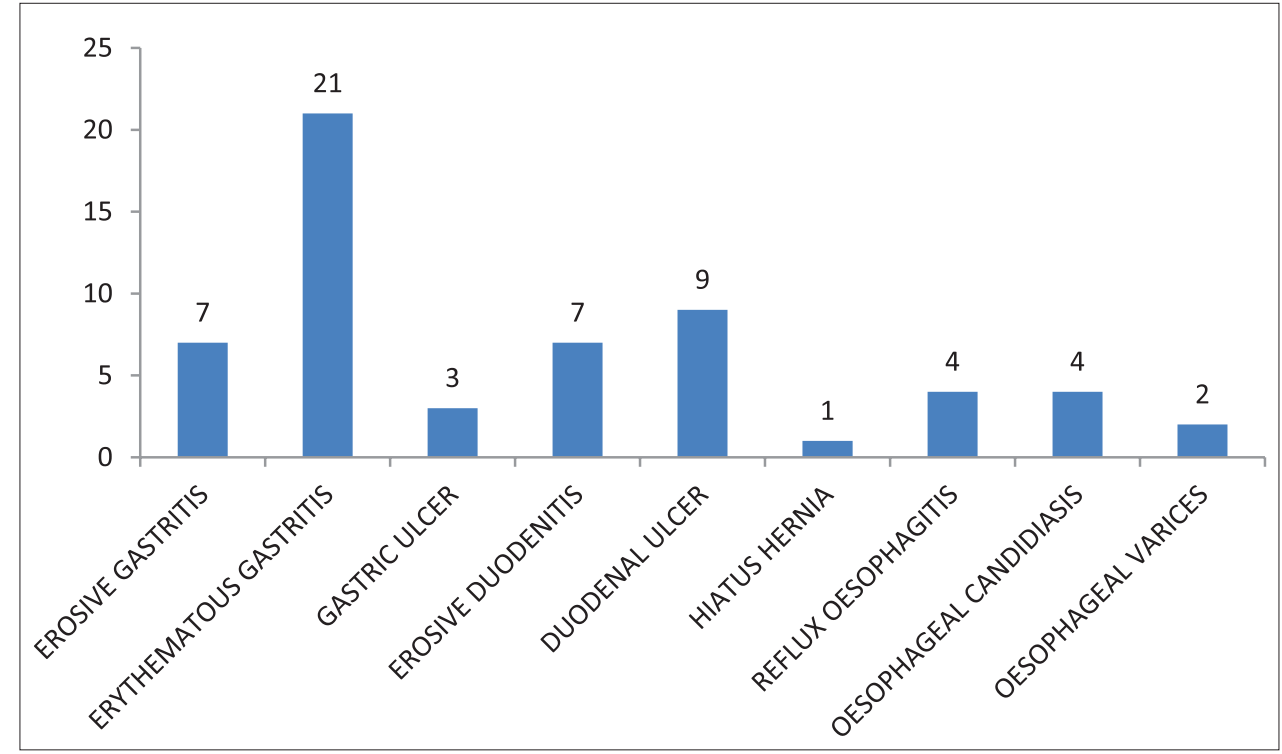

Figure 4: H.pylori and endoscopic findings

Mahadeva and Goh have reported a global prevalence of uninvestigated dyspepsia and functional dyspepsia between $7 \%$ to $45 \%$ and $11 \%$ to $29.2 \%$ respectively. ${ }^{4}$ According to Grainger et al, the prevalence of dyspepsia from published figures vary from $20 \%$ to $40 \% .^{5}$ A study from Mumbai by Shah et al reported dyspepsia in one-third of the population. Of these, $12 \%$ had significant symptoms and $40 \%$ received treatment. ${ }^{6}$

In our study, the mean age was 42 (SD \pm 14 ) years, of which $54 \%$ of the subjects belonged to the age group of 20 to 40 years, $35 \%$ between 41 to 60 years and the remaining $11 \%$ above 61 years of age. $61 \%$ of our subjects were males and 39\% were females. The type of dyspeptic symptoms did not vary between males and females. Jones and Lydeard noticed a decrease in frequency of symptoms with age, especially in males. ${ }^{7}$ Talley et al, following a questionnaire study conducted in Minnesota, have reported higher incidence of dyspepsia among younger age groups and females. ${ }^{8}$

In our study, no significant difference was noticed in dyspeptic symptoms among different occupational groups. But Sonnenberg and Haas have reported 
higher incidence of gastric and duodenal ulcer and non-ulcer dyspepsia among manual labourers in German population. ${ }^{9}$

Majority of our subjects were non vegetarians $(83 \%)$, but no relation was found between diet and dyspeptic symptoms. Similar results were also obtained in a case control study by Cuperus et al. ${ }^{10}$ Another case control study from Norway by Lind et al concluded that abdominal symptoms were due to food hypersensitivity. ${ }^{11}$ Alcohol intake and smoking were reported in $43 \%$ and $38 \%$ of our patient, respectively. Shah et al also found higher prevalence of dyspepsia among alcoholics and smokers. ${ }^{6}$ But Talley et al did not find smoking and alcohol to be important risk factors with respect to dyspepsia. ${ }^{8}$ NSAID consumption was reported in $31 \%$ of our patients. Studies have shown that NSAID can provoke dyspepsia, and the incidence was less with COX-2 inhibitors. ${ }^{12,13}$ But such a relation was not noticed by Talley et al. ${ }^{8}$

Epigastric pain and epigastric burning sensation were the main symptoms among our patients. Shah et al found abdominal fullness, abdominal pain, heartburn and belching as the most common symptoms in their study. ${ }^{6}$

Majority of our subjects had findings of erythematous and erosive gastritis on endoscopy ( $42 \%$ and $21 \%$ respectively). They were of mild to moderate severity. Erythematous gastritis was mainly found in the corpus of stomach, followed by antrum and fundus; while erosive gastritis was confined to the antrum. Duodenal ulcers were more common among men. Duodenitis and duodenal ulcers were the main findings among alcoholics. Normal endoscopic findings were seen in $18 \%$ of our patients. Eman et al, in their analysis of dyspeptic Kuwaiti patients, observed normal study in $32 \%$. Other findings included non-erosive antral gastritis $(26 \%)$, duodenitis $(17.5 \%)$, duodenal ulcer $(11.5 \%)$, deformed bulb (4\%), esophagitis (7\%) and erosive gastritis $(2 \%) .{ }^{14}$

Graham et al in their study on asymptomatic population of USA, reported $\mathrm{H}$. pylori infection in $52 \%$ of their subjects; and their incidence increased with age at a rate of $1 \%$ per year. The frequency was higher in blacks than whites. ${ }^{15}$ Eman et al noticed H. pylori in $88.5 \%$ of their patients. ${ }^{14}$ A study on South Indian population (Kerala) by Adlekha et al revealed a 62\% prevalence of H. pylori. ${ }^{16}$ In our study, the presence of H. pylori was $35 \%$; with prevalence being more among alcoholics. The low incidence in our study could be due to the use of only a single method (RUT) to detect H. pylori. Yet another reason might be the overall improvement in sanitation and hygiene. Decreasing trend in $\mathrm{H}$. pylori infection was also noticed by Xia et al. ${ }^{17}$

\section{CONCLUSION}

The mean age of dyspeptic patients in our study was 42 $(\mathrm{SD} \pm 14)$ years. Majority of the patients belonged to younger age group (20 to 40 years). The patients were predominantly males. Homemakers and those leading a retired life were the main victims. Smoking, alcohol and NSAID consumption were contributory factors. Epigastric pain and epigastric burning sensation were the main presenting symptoms. Erythematous gastritis followed by erosive gastritis were the common endoscopic findings. Duodenitis and duodenal ulcers were more among alcoholics. Some of the patients had normal findings on endoscopy. H. pylori infection was seen in more than one fourth of our patients.

\section{REFERENCES}

1. Jan Tack. Dyspepsia. In: Feldman M, Friedman LS, Brandt LJ eds. Sleisenger and Fordtran's Gastrointestinal and Liver Disease, 10th ed. Saunders Elsevier, 2015; pp 194-206.

2. Baron JH and Sonnenberg A. Early History of Dyspepsia and Peptic Ulcer in the United State.Am J Gastroenterol 2009; 104(12): 2893- 2896.

3. El-Serag HB and Talley NJ. Systemic review: the prevalence and clinical course of functional dyspepsia. Aliment Pharmacol Ther 2004; 19(6):643-654.

4. Mahadeva S and Goh KL. Epidemiology of functional dyspepsia: a global perspective. World J Gastroenterol 2006; 12(17):26612666.

5. Grainger SL, Klass HJ, Rake MO and Williams JG. Prevalence of dyspepsia: the epidemiology of overlapping symptoms. Postgrad Med J 1994; 70(821):154-161.

6. Shah SS, Bhatia SJ and Mistry FP. Epidemiology of dyspepsia in the general population in Mumbai. Indian J Gastroenterol 2001; 20(3):103-106.

7. Jones R and Lydeard S. Prevalence of symptoms of dyspepsia in the community. BMJ 1989; 298(6665):30-32.

8. Talley NJ, Zinsmeister AR, Schleck CD and Melton LJ. Smoking, alcohol, and analgesics in dyspepsia and among dyspepsia subgroups: lack of an association in a community. Gut 1994; 35(5): 619-624.

9. Sonnenberg A and Haas J. Joint effect of occupation and nationality on the prevalence of peptic ulcer in German workers. Br J Ind Med 1986; 43(7):490-493.

10. Cuperus P, Keeling PW and Gibney MJ. Eating patterns in functional dyspepsia: a case control study. Eur J Clin Nutr 1996; 50(8):520-523.

11. Lind R, Olafsson S, Hjelland I, Berstad A and Lied GA. Lifestyle of patients with self-reported food hypersensitivity differ little from controls. Gastroenterol Nurs 2008; 31(6):401-410.

12. Pavelka K, Recker DP and Verburg KM. Valdecoxib is as effective as diclofenac in the management of rheumatoid arthritis with a lower incidence of gastroduodenal ulcers: results of a 26-week trial. Rheumatology (Oxford). 2003; 42(10):12071215.

13. Langman MJ, Jensen DM, Watson DJ, Harper SE, Zhao PL, Quan $\mathrm{H}$, et al. Adverse upper gastrointestinal effects of rofecoxib compared with NSAIDs. JAMA 1999; 282 (20):1929-1933.

14. Abahussain EA, Hasan FA and Nicholls PJ. Dyspepsia and 
Helicobacter pylori infection: Analysis of 200 Kuwaiti patients referred for endoscopy. Annals of Saudi Medicine 1998; 18(6):502-505.

15. Graham DY, Malaty HM, Evans DG, Evans DJ, Klein PD and Adam E. Epidemiology of Helicobacter pylori in an asymptomatic population in the United States. Effect of age, race, and socioeconomic status. Gastroenterology 1991; 100(6):14951501.

16. Adlekha S, Chadha T, Krishnan P and Sumangala B. Prevalence of Helicobacter Pylori Infection Among Patients Undergoing Upper Gastrointestinal Endoscopy in a Medical College Hospital in Kerala, India. Annals of Medical and Health Sciences Research 2013; 3(4):559-563.

17. Xia B, Xia HH, Ma CW, Wong KW, Fung FM, Hui CK, et al. Trends in the prevalence of peptic ulcer disease and Helicobacter pylori infection in family physician-referred uninvestigated dyspeptic patients in Hong Kong. Aliment Pharmacol Ther 2005; 22(3):243-249.

Authors Contribution:

RGM - Concept and design of case report, reviewed the literature, manuscript preparation and critical revision of manuscript; and corresponding author; TA - Critical revision of manuscript.

Source of Support: Nil, Conflict of Interest: None declared. 